\title{
Energy Efficiency Contours for Single-Carrier Downlink Channels
}

\author{
Amir Akbari, Student Member, IEEE, Muhammad A. Imran, Rahim Tafazolli, and Mehrdad \\ Dianati, Member, IEEE,
}

\begin{abstract}
Energy efficiency has become an important aspect of wireless communication, both economically and environmentally. This letter investigates the energy efficiency of downlink AWGN channels by employing multiple decoding policies. The overall energy efficiency of the system is based on the bits-per-joule metric, where energy efficiency contours are used to locate the optimal operating points based on the system requirements. Our novel approach uses a linear power model to define the total power consumed at the base station, encompassing the circuit and processing power, and amplifier efficiency, and ensures that the best energy efficiency value can be achieved whilst satisfying other system targets such as QoS and rate-fairness.
\end{abstract}

Index Terms-Broadcast Channel, Capacity Region, Contour, Downlink, Energy Efficiency.

\section{INTRODUCTION}

$\mathbf{T}$ HERE are two motivating factors for the recent awareness in energy efficiency (EE) of wireless networks. Firstly, to address the environmental impact by reducing carbon emissions, and secondly from an operators point of view, to increase profits by reducing electricity bills. With the exponential growth of mobile communications and increasing demand for multi-media services, the increase in the data traffic will ultimately be a dominating factor and unless new energy saving strategies are employed, huge limitations will have to be enforced on data usage.

The fundamental results of energy-efficient point to point links can be traced back to [1], where the capacity in bitsper-joule is given for a single link on frequency selective channels. The same metric was employed in our previous work [2], where the average EE of Additive White Gaussian Noise (AWGN) broadcast channels (BC) where investigated under Time-division (TD), Frequency-division (FD) and Superposition coding (SPC) [3].

In this letter, the overall system $\mathrm{EE}$ of a multi-user $\mathrm{BC}$ is investigated under SPC, where a linear power model is used to define the total power consumed at the base station (BS), taking into account the circuit power, processing power and amplifier efficiency. The system EE will be measured using two different decoding policies, where a framework is used to choose the optimal decoding policy for each rate point such that EE is maximized. EE contours which we introduced in [4] will then be used to identify optimal operating points based

Manuscript received July 12, 2011. The associate editor coordinating the review of this letter and approving it for publication was G. Mazzini.

The authors are with the Centre for Communication Systems Research, University of Surrey, Guildford, Surrey, GU2 7XH, UK (e-mail: a.akbari@surrey.ac.uk) on the rate-fairness and quality of service (QoS) requirement of the system.

\section{SYSTEM DESIGN}

A single-carrier downlink channel with $K$ receivers is considered, where the received signal is corrupted by AWGN with power spectral density $N_{0} / 2$. The system bandwidth is set to $B$, and the channel power gain from the transmitter to the $k^{t h}$ receiver is denoted as $g_{k}$, where users are ordered relative to their channel gains such that $g_{1} \leq g_{2} \leq \ldots \leq g_{K}$. The transmit power of user $k$ is denoted as $p_{k}$, and the total transmit power of the BS is set to $P$ such that $P=\sum_{k=1}^{K} p_{k}$.

Therefore, the rate of user $k$ is given by the well-known Shannon formula [5] as

$$
R_{k} \leq B \log _{2}\left(1+\frac{p_{k} g_{k}}{N_{0} B}\right), \quad k=1,2, \ldots, K
$$

EE is evaluated through the bits-per-joule metric, therefore, the overall EE of the multi-user downlink system is defined as the total transmission rate over the total power consumed at the BS.

$$
E E_{\mathcal{T}}=\frac{\sum_{k=1}^{K} R_{k}}{P_{T}}
$$

A linear relation is modeled between the total power consumption and radiated power of the BS [6] such that

$$
P_{T}=P_{c}+\beta P
$$

where, $P_{c}$ and $\beta$ denote the circuit and processing power, and amplifier efficiency coefficient respectively.

\section{Multiple Decoding Policies}

Two decoding orders are employed, where $\pi$ is defined as the permutation of the ordered sequence such that $\pi(k)$ is the $k^{\text {th }}$ element of the permutation. Assuming the channel gains are ordered as discussed above, the following two decoding policies are considered:

Decoding Policy 1: User 1 decoded last, therefore achieving its single user bound, whilst other users get a non-zero rate.

$$
\mathcal{P}_{1}: \pi=\{K, K-1, \ldots, 1\}
$$

Decoding Policy 2: User $K$ decoded last, therefore achieving its single user bound, whilst other users get a non-zero rate.

$$
\mathcal{P}_{2}: \pi=\{1,2, \ldots, K\}
$$




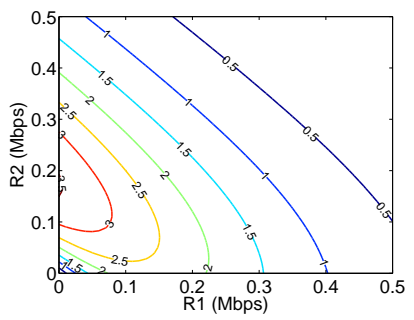

(a) Decoding Policy 1

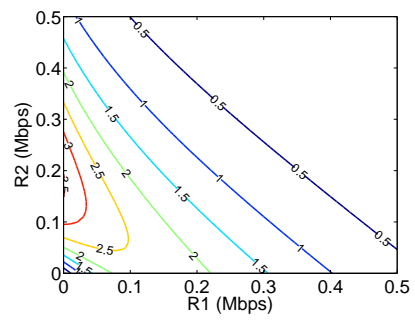

(b) Decoding Policy 2
Fig. 1. Energy efficiency contours using decoding policy 1 and 2

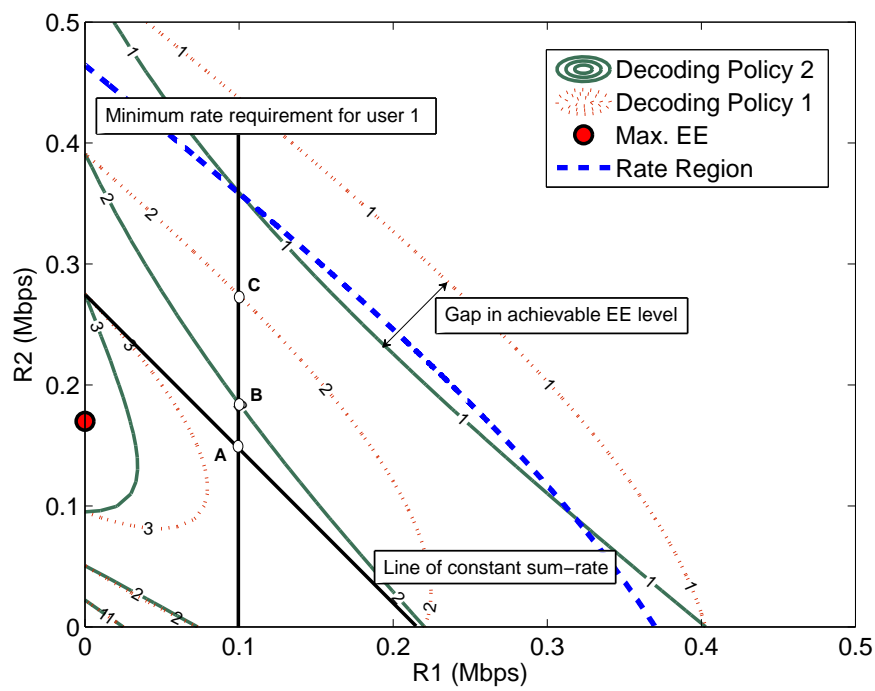

Fig. 2. Comparison of energy efficiency contours using different decoding policies (Data on contours define energy efficiency value in Mbits/Joule)

By employing SPC with either decoding policy, the user decoded last can achieve its single user bound while other users experience some finite multi-user interference.

Therefore, the transmission rate of user $k$ can be shown as:

$$
R_{\pi(k)}=B \log _{2}\left(1+\frac{p_{\pi(k)} g_{\pi(k)}}{N_{0} B+g_{\pi(k)} \sum_{j=1}^{k-1} p_{\pi(j)}}\right)
$$

Moreover, the transmit power of user $k$ can be expressed as:

$$
p_{\pi(k)}=\left(2^{\frac{R_{\pi(k)}}{B}}-1\right)\left(N_{0} B g_{\pi(k)}^{-1}+\sum_{j=1}^{k-1} p_{\pi(j)}\right)
$$

For each rate point, (2) and (6) are used to obtain the corresponding $\mathrm{EE}$ value. The collection of all rate points leading to the same EE value define an EE contour.

\section{System Evaluation}

This section presents the EE contours of the downlink channel. To simplify the exposition of concept and ease of graphical representation, a 2-user case is considered here, but the general properties and performance measures hold for $K>2$. The system is designed such that, $g=[0.004,0.008]$, $B=100 \mathrm{KHz}, N_{0}=10^{-9} \mathrm{~W} / \mathrm{Hz}, P_{c}=0.02$ Watts and $\beta=$ 1 (unless stated otherwise).

\section{A. EE Contours with Multiple Decoding Policies}

Fig.1 shows the EE contours of the system using both decoding policies. It is clearly seen that maximum EE is achieved by only transmitting to the user with highest channel gain (user 2 in this case), regardless of the decoding policy employed. Therefore the maximum achievable $E E_{\mathcal{T}}$ will be the same using either decoding policy.

The EE contours in Fig.1 demonstrate one of the most fundamental differences between energy-efficient design in the uplink and downlink, where it was shown in [4] that, maximum EE is achieved in the uplink when, all users transmit simultaneously by employing a successive interference cancellation (SIC) receiver.

Although the same maximum achievable $E E_{\mathcal{T}}$ is obtained using both decoding policies, the shape of the contours are different, indicating that different rate-pairs would be achievable. To demonstrate this difference, both EE contours have been plotted in Fig.2, where the downlink rate region is also shown based on a maximum transmit power constraint of 300 $\mathrm{mW}$. The gradient of the EE contours for decoding policy 2 are much steeper, therefore, the gap between different EE levels are less compared to the contours from decoding policy 1.

As an example, consider the case when decoding policy 1 is used, and a target EE of $1 \mathrm{Mbits} / \mathrm{Joule}$ is required. As it can be seen in Fig.2, this contour is outside of the rate region, and hence not achievable. However, the same target EE value can be achieved by employing decoding policy 2 .

The second main difference between the two decoding polices arises when one user has a minimum rate requirement and the systems target is to maximise the EE whilst keeping the sum-rate constant. Assuming that the minimum rate requirement of User 1 is $0.1 \mathrm{Mbps}$, the intersection of the line of constant sum rate (line with a slope of -1) and the minimum rate requirement of User 1 gives the optimal operating point, which is marked as point $\mathbf{A}$ in Fig.2. It should be noted that at this point, a higher EE is achieved when decoding policy 1 is employed.

Another aplicable scenario is when one user has a minimum rate requirement whist the system has an EE target. Assuming that the minimum rate requirement of User 1 is $0.1 \mathrm{Mbps}$, and a system EE target of $2 \mathrm{Mbits} / \mathrm{Joule}$ is required. The intersection of the minimum rate line with the desired contour gives the optimal operating points, which is shown as point $\mathbf{B}$ for decoding policy 2 and point $\mathbf{C}$ for decoding policy 1. Both decoding policies can meet the requirements of the system but employing decoding policy 1 will allocate a higher rate to user 2 (the stronger user).

\section{B. Optimality Measures}

Let $E E_{\mathcal{T}}^{\mathcal{P}_{1}}$ and $E E_{\mathcal{T}}^{\mathcal{P}_{2}}$ denote scenarios where decoding policy 1 and 2 have been chosen respectively. For each ratevector, the decoding policy that achieves the highest $\mathrm{EE}$ is chosen

$$
E E_{\mathrm{OPT}}=\max \left(E E_{\mathcal{T}}^{\mathcal{P}_{1}}, E E_{\mathcal{T}}^{\mathcal{P}_{2}}\right)
$$

The resulting contours are shown in Fig.4, which are identical to the contours obtained using decoding policy 1 . Several 


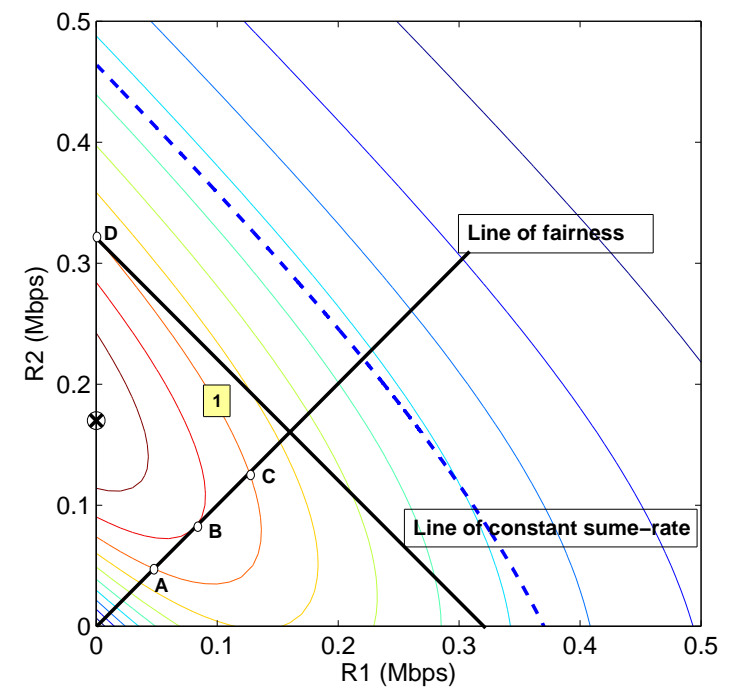

Fig. 3. Energy efficiency contours using $E E_{\mathrm{Opt}}$

optimality measures will be considered as discussed below:

\section{1) Maximizing $E E$}

In scenarios where the only aim of the system is to maximize the overall $\mathrm{EE}$, the optimal rate pair is chosen such that $E E_{\mathrm{Opt}}$ is maximized, and is marked with a cross in Fig.3. As shown, maximum EE is achieved by only transmitting to the strong user.

\section{2) Maximizing EE with Rate-fairness}

Points with equal rate share for all users lie on a line passing through the origin with a slope of +1 (line of fairness). Therefore, the maximum achievable energy-efficient rate-fair point will be the point at which the line of fairness tangentially touches the EE contours, shown as point $\mathbf{B}$.

\section{3) Achieving Target EE with Maximum Sum-rate}

For a fixed EE target, the corresponding contour is of interest to the system. The point on the desired contour (contour labeled 1) tangentially touching the line of constant sum-rate will give the rate pair achieving the target EE and having the maximum possible sum-rate, and is shown as point D.

\section{4) Achieving Target EE with Rate-fairness}

The intersection of the line of fairness and the desired EE target will give the optimal rate pair for this criterion. Here, there are two intersections (points $\mathbf{A}$ and $\mathbf{C}$ ), with both meeting the required criteria in terms of fairness and EE, however, point $\mathbf{C}$ has a higher sum-rate.

\section{Effect of $\beta$ on Energy Efficiency}

The power amplifier coefficient $(\beta)$ is referred to the ratio of the direct input power to the transmit power [6]. Efficient power amplifiers provide better circuit performance and require less cooling, thus increasing the EE of BSs. The results in Fig. 4 show the effect of $\beta$ on the $\mathrm{EE}$ of the BC.

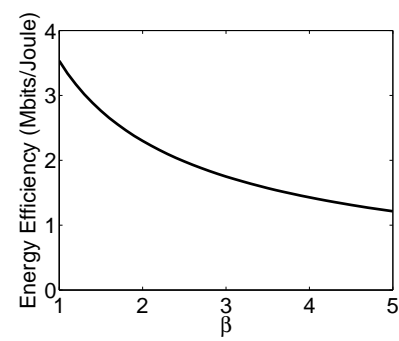

(a) Effect of $\beta$ on $E E_{\mathrm{Opt}}$

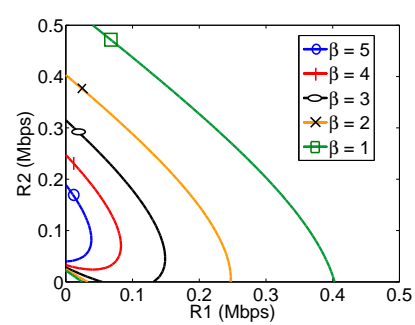

(b) EE fixed to 1 Mbits/Joule
Fig. 4. Effect of amplifier efficiency coefficient on energy efficiency

Fig.4 (a) shows the decrease in the maximum achievable EE of the system when $\beta$ is increased. Therefore, a higher $\beta$ translates into a less energy-efficient power amplifier.

Fig.4 (b) demonstrates the same finding using EE contours. The system EE has been set to 1 Mbits/Joule, with different amplifier efficiency coefficients. The EE contours show that, as $\beta$ is increased, the size of the contour becomes smaller, resulting in lower transmit rates for both users. This is extremely important in scenarios where users have a minimum rate requirement and $\mathrm{EE}$ target to meet.

\section{CONCLUSION}

In this letter, we use EE contours to ensure that the best EE value can be achieved while satisfying other system targets such QoS and fairness. Our novel approach uses a linear power model to calculate the overall consumed power at the BS, where the transmit power, circuit power and amplifier efficiency coefficient are incorporated in the system design. Numerical results provide a detailed analysis of the effects of multiple decoding policies and amplifier efficiency coefficient on the total EE of the multi-user BC.

\section{REFERENCES}

[1] H. M. Kwon, T. G. Birdsall, "Channel Capacity in Bits per Joule," IEEE Journal of Oceanic Engineering, vol. 0E-11, no. 1, pp.97-99, Jan. 1986.

[2] A. Akbari, M. A. Imran, and R. Tafazolli, "Maximizing Average Energy Efficiency for Two-user AWGN Broadcast Channel," proceedings of IEEE Future Network \& Mobile Summit, Poland, June 2011.

[3] A. Goldsmith, Wireless Communications. Cambridge University press, 2005 .

[4] A. Akbari, M. A. Imran, R. Hoshyar, A. Amich, and R. Tafazolli, "Average Energy Efficiency Contours with Multiple Decoding Policies," IEEE Communications Letter, vol. 15, no. 5, pp. 506-508, May 2011.

[5] C. E. Shannon and W. Weaver, A Mathematical Theory of Communication. Urbana, IL: University Illinois Press, 1949.

[6] A. J. Fehske, P. Marsch, and G. P. Fettweis, "Bit per joule efficiency of cooperating base stations in cellular networks," 3rd International Workshop on Green Communications, IEEE Global Communications Conference, GLOBECOM 2010, Florida, Dec. 2010. 